\title{
Is reproduction of endemic plant species particularly pollen limited in biodiversity hotspots?
}

\author{
Conchita Alonso, Jana C. Vamosi, Tiffany M. Knight, Janette A. Steets and Tia-Lynn Ashman \\ C. Alonso (conalo@ebd.csic.es),Dept of Evolutionary Ecology, Estación Biológica de Doñana, CSIC, Apdo. 1056, ES-41080 Sevilla, \\ Spain. - J. C. Vamosi, Dept of Biological Sciences, Univ. of Calgary, 2500 University Drive NW, Calgary, AB, T2N 1N4, \\ Canada. - T. M. Knight, Dept of Biology, Washington Univ., Box 1137, St. Louis, MO 63130, USA. - J. A. Steets, Dept of Botany, Oklahoma \\ State Univ., 104 Life Sciences East, Stillwater, OK 74078-3013, USA. - T.-L. Ashman, Dept of Biological Sciences, Univ. of Pittsburgh, \\ Pittsburgh, PA15260, USA.
}

\begin{abstract}
Current evidence suggests that plants in biodiversity hotspots suffer more from pollen limitation of reproduction than those in lower diversity regions, primarily due to the response of self-incompatible species. Species in biodiversity hotspots may thus be more at risk of limited reproduction and subsequent population decline. Should these species have restricted ranges (i.e. be endemics to a certain region), pollen limitation within highly diverse regions may pose an important threat to global plant biodiversity. We further dissect the global pattern by exploring whether pollen limitation of range-restricted (endemic) species is distinctive and/or relates differently to species diversity than that of widespread (non-endemic) species. To provide a preliminary test of this prediction we conducted both cross-species and comparative phylogenetic meta-analyses to determine the effect of endemism on the magnitude of pollen limitation and its relationship with regional species richness. Our data set included 287 plant species belonging to 78 families distributed world-wide. Our results revealed that endemism and self-compatibility contribute to the global association between pollen limitation and species richness. Self-incompatible species were more pollen limited than self-compatible ones, and the PICs analysis indicated that transitions to endemism were associated with transitions to self-compatibility. The relationship between pollen limitation and species richness was significant only for the self-incompatible species, and was monotonically increasing in non-endemic species but accelerating in the endemic species. Thus, self-incompatible endemic species from biodiversity hotspots are at the greatest risk of pollination failure, a previously unknown aspect suggesting this group of species as a top priority for future development of conservation strategies. In contrast, reproduction of self-compatible species appears to be unrelated to plant diversity, although we caution that current data do not account for the reproductive limitation due to the quality of pollen received. Understanding the mechanisms underlying these patterns requires further investigation into plant-plant pollinator mediated interactions and the dynamics of pollen transfer in communities differing in species diversity.
\end{abstract}

Pollen limitation of plant reproduction is a widespread phenomenon (reviewed by Ashman et al. 2004). Ecological disturbances such as decline of pollinators, habitat fragmentation, introduction of alien species, and climate change may increase pollen limitation (Knight et al. 2005, Memmott et al. 2007). This phenomenon has direct consequences for human welfare because fruit or seed production from $76 \%$ of the 115 leading global food crops is dependent upon animal pollination (Klein et al. 2007). Pollen limitation also poses challenges for conservation of wild flora because most plants threatened by extinction occur in small or sparse populations as well as in fragmented or specialized habitats, characteristics that are expected to amplify the risk of pollination failure (Wilcock and Neiland 2002). In fact, in the wild flora, a significant positive relationship between pollen limitation and species richness was found at the global scale (Vamosi et al. 2006) - a pattern that deserves further analysis because many rare, globally-threatened and endangered species inhabit the most species rich areas on earth (Myers et al. 2000). One functional explanation of this pattern is that plants occurring in species-rich communities experience greater interspecific competition for pollinators than those in species-poor communities (Vamosi et al. 2006). Otherwise, environmental factors related to variation in regional species richness on a global scale, such as high elevation and cold weather (Mutke and Barthlott 2005, Ohlemüller et al. 2008), can directly correlate with pollen limitation and generate such pattern. Another non-exclusive possibility unexplored so far is that the occurrence of range-restricted species (hereafter referred to as endemics),

The review of and decision to publish this paper has been taken by the above noted SE. The decision by the handling SE is shared by a second SE and the EiC. 
which are often restricted to rare local climates (Ohlemüller et al. 2008), is higher in communities with greater species richness and contributes to the higher level of pollen limitation observed.

Why would endemism affect pollen limitation? We predict that endemics might be more pollen limited because 1) in the few cases in which they have been compared within a given region endemics differ from widespread species in many plant features related to the attraction of, and reliance on, pollinators for reproduction (Kruckeberg and Rabinowitz 1985, Kunin and Shmida 1997, Lavergne et al. 2004), some of which (e.g. fewer and smaller flowers) are associated with increased pollen limitation (Knight et al.2005); 2) endemics also have smaller population sizes, reduced density and/or stronger habitat specificity than widespread species (Rabinowitz 1981, Lavergne et al. 2004, Thompson et al. 2005) features that can also increase pollen limitation (Knight et al. 2005); 3) endemics tend to produce significantly fewer seeds than widespread species (Murray et al. 2002, Lavergne et al. 2004, Thompson et al. 2005) potentially indicating pollen limitation of seed production; and 4) a small number of direct comparisons between endemic and non-endemic congeners found stronger pollen limitation in the endemics (Karron 1987, Rymer et al. 2005). Taken together, these points raise the possibility that endemics are poor competitors for pollinators within their communities and, given the higher prevalence of endemism in species rich regions (Myers et al. 2000), drive the positive relationship between pollen limitation and species richness. In addition, endemics may suffer more from quality aspects of pollen limitation relative to more widespread species owing to features such as low genetic diversity or lack of compatible mates associated with small population sizes (Byers 1995, Cole 2003, Kirchner et al. 2005). However, if endemics tend to be self-compatible and have traits that promote autonomous self-pollination (Krukeberg and Rabinowitz 1985, Kunin and Shmida 1997, Lavergne et al. 2004) then they may be buffered against increasing interspecific competition for pollinators. In this case, pollen limitation of endemics would not vary with changes in community diversity.

To provide a preliminary test of these predictions we conducted meta-analyses of published studies of pollen limitation. We employed both cross-species (TIPS) and phylogenetically corrected (PICs) meta-analytical approaches, both of which have been used to analyze the association between pollen limitation and plant traits (Larson and Barrett 2000, Knight et al. 2005, Vamosi et al. 2006). In doing so, we sought to answer the following questions:

1. Are studies of pollen limitation on endemic species more often conducted in regions with high species richness?

2. Do endemic species exhibit higher pollen limitation than non-endemic species? Does the answer depend on self-compatibility?

3. Does the relationship between pollen limitation and regional species richness differ for endemic and nonendemic species at the global scale? If so, is it consistent across the whole range of species diversity (i.e. is the relationship monotonically increasing or accelerating)?

\section{Methods}

\section{Data set}

For our study we started with the data set (241 species from studies published between 1981-2003) used by Vamosi et al. (2006), and updated it by adding data from studies published between 2004 and 2007. We searched ISI Web of Science using the same key words used to create the base data set (pollen limit*, supplement* poll*, hand poll*, Knight et al. 2005). In addition, we searched specifically for studies on endemic species using the key words endemi* and pollinat* across the entire span of publication years 1981-2007. Altogether we obtained data on pollen limitation for 299 species, most of which were represented by a single location. In the seven cases for which a species had been evaluated in two locations that differed in species richness, we included the data for both studies as independent data points, thus the initial data set had 306 entries. Pollen limitation estimates were based on fruit set (fruits/flowers) of plants given supplemental pollen compared to those receiving ambient pollen loads. Fruit set was the most commonly measured response variable reported in the literature, and it is a reasonably good indicator of pollen limitation in the number of seeds produced by the entire plant, arguably the best estimate of female fitness (Knight et al. 2005). We used author's descriptions of their study systems and several web sources to determine geographic ranges. While including geographic range size as a quantitative variable would be ideal, detailed species range maps are not currently available for most of the species in our dataset. Thus, we reduced our range variable to a categorical one, based on species descriptions as either: 1 ) range-restricted species, i.e. those confined to a geographically restricted region (e.g. Sonoran desert, Florida, Cape region, Iberian Peninsula) - we refer to these as 'endemics'; and 2) species that do not meet the criteria for group (1) - we refer to these as 'non-endemics'. We were not able to confidently characterize the geographic range of six species and thus, these were eliminated from further analysis. Local population size and habitat specificity, other important aspects of plant rarity (sensu Rabinowitz 1981) that may also affect pollen limitation could not be quantified for most of the species in our dataset, and thus were not strictly analysed. Only the effects of the local size of the study population could be coarsely explored. To do so, we distinguished between large and small populations based on the author's (largely qualitative) descriptions of their study systems. Given the importance of self-compatibility in propensity for pollen limitation (Knight et al. 2005), and for the association between species richness and pollen limitation (Vamosi et al. 2006), we also recorded whether each species was self-compatible or selfincompatible. We could not determine self-compatibility of six species; consequently these species were excluded from analyses. The final data set had 294 entries, with 287 species belonging to 78 families, half of the families being represented by a single study species (Fig. 1; see Supplementary material Appendix 1 for species list and endemism classification).

Following Knight et al. (2005), the magnitude of pollen limitation was calculated as the natural log response ratio ( $\ln \mathrm{R})$, where $\mathrm{R}$ is the ratio between the mean proportion fruit set (fruit/flower) of plants receiving supplemental and ambient pollen loads. An estimate of regional plant species 


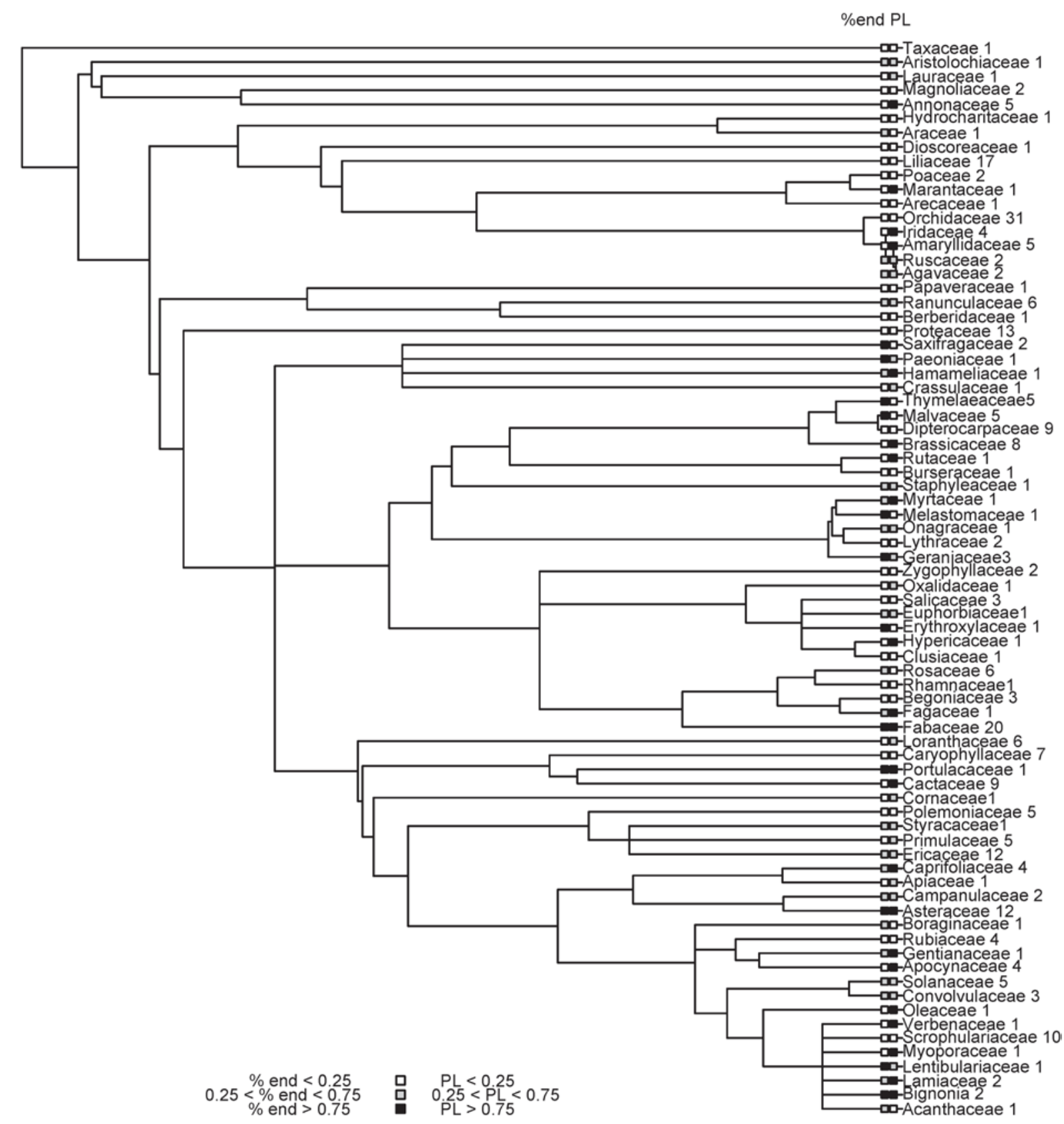

Figure 1. Summary phylogenetic tree showing the frequency of endemism and magnitude of pollen limitation in the 287 study species mapped onto the phylogeny of plant families. Grey scale in the two boxes indicate the three quantiles (low: $<0.25$, mid-range: $0.25><0.75$, high: $>0.75$, respectively) for percent endemic species per family (' $\%$ end') and the average pollen limitation effect size for the species in that family ('PL'), respectively. Numbers indicate the number of study species per family. Only pollen limitation exhibited phylogenetic signal.

richness at the standard area size of $10000 \mathrm{~km}^{2}$ was obtained from BIOMAPS (Barthlott et al. 1999) and log-transformed $[\log (\mathrm{Sr})]$ before analysis to reduce heteroscedasticity.

\section{TIPS statistical analyses}

TIPS analyses treated species as independent data points and thus would largely reflect the ecological relationships of pollen limitation among contemporary species. Unless otherwise stated, analyses were performed using the SAS statistical package (SAS Inst. 2002). We used $x^{2}$-tests to evaluate the association between endemism and self-compatibility in our data set. We performed a non-parametric Kruskal-Wallis test to determine whether endemic species were studied in regions with higher species richness than non-endemics.

To test whether endemic species exhibited higher pollen limitation than non-endemic species, we used a two-way ANOVA. The model took into account the effect of endemism and self-compatibility as well as the potential interaction between both effects, the estimation of the covariance parameters was performed by residual maximum likelihood (REML). 
We used non-parametric regression to assess the shape of the relationship between pollen limitation and regional species richness in endemics and non-endemics. In non-parametric regression, no assumptions are made regarding the specific form of the function linking the dependent and independent variable, other than that it is smooth. Cubic splines were fitted to the data that minimized a generalized cross-validation score, as described by Schluter (1988) and implemented in software GLMS ver. 4.10 (<www.zoology.ubc.ca/ schluter/software. $\mathrm{html}>$ ). Confidence intervals of fitted regressions were obtained by bootstrapping (1000 times). Because self-compatible and self-incompatible species showed contrasting relationships in the base data set (Vamosi et al. 2006), we analysed endemic and non-endemic species separately by self-compatibility.

\section{PICs statistical analyses}

We also adopted a phylogenetic meta-analytic approach, taking into account the evolutionary relationships among the study species. A hypothesized phylogenetic tree for the species included in our data set was obtained by using the maximally resolved supertree in PHYLOMATIC (<www.phylodiversity. net/phylomatic $>$ ). This supertree is based on the hypothesized phylogenetic relationships of angiosperm families according to the APG taxonomy (APGII), modified and updated by Stevens (2008). Branch lengths were calibrated from the minimum age of clade divergence from Wikström et al. (2001) using the BLADJ function within PHYLOMATIC. To assess whether the response variable (pollen limitation) and the explanatory traits studied (endemism, self-compatibility and regional species richness) show phylogenetic association within our data set we used the lambda $(\lambda)$ parameter (Freckleton et al. 2002). If $\lambda$ is significantly different from zero, this indicates the presence of a phylogenetic signal, where closely related species are similar with regard to a particular trait. Phylogenetic independent contrasts (PICs) were calculated using the software application PHYLOCOM (Webb et al. 2008). To assess whether binary traits (such as endemism or self-compatibility) were correlated with higher pollen limitation, we analyzed the PICs in pollen limitation effect size with every transition to the trait of interest (e.g. endemism) with one-tailed Wilcoxon signed rank tests. Because it is not yet straightforward to perform phylogenetically-correct multivariate statistics, we separated our dataset into self-incompatible and self-compatible species. Within each subset we repeated our examinations of associations between endemism and pollen limitation. To analyze the relationships between species richness and pollen limitation, we regressed the contrasts in pollen limitation against the contrasts in regional species richness, forcing the regression through the origin (Garland et al. 1992), and also examined whether the relationship between species richness and pollen limitation was stronger in endemics than non-endemics.

\section{Results}

\section{Characterization of the data set}

\section{Phylogenetic signal}

Our analyses revealed that the magnitude of pollen limitation exhibit a strong phylogenetic signal $(\lambda=0.45, p=0.0006$;
Fig. 1), indicating that close relatives were more similar in pollen limitation level than expected by chance. Among the explanatory variables analyzed only endemism did not show phylogenetic signal in our data set $(\lambda<0$. 0005, $p=1)$, while the other traits did exhibit a strong phylogenetic signal indicating that closely-related species are more similar in selfcompatibility $(\lambda=0.23, p=0.001)$, and species richness of their communities $(\lambda=0.82, p<0.0001)$ than expected by chance.

\section{Endemism and local population size}

Based on our classification of endemism, 31\% of the species in our dataset were endemic. Furthermore, our coarse classification of species population size distinguished 224 species with large populations and 63 species with small populations. As is often observed, endemism and local population size were related (Rabinowitz 1981). In fact, $53 \%$ of the endemic species in our data set were characterized by small local population sizes. This contrasts with only $8 \%$ of the non-endemic species being classified as having small local population sizes.

\section{Geographic distribution}

The study species were located in regions that cover the whole range of global diversity zones described by Barthlott et al. (1999), with a $\log (\mathrm{Sr})$ that ranged from 2 to 3.78, which is equivalent to a range of 100-6000 vascular plant species per $10000 \mathrm{~km}^{2}$ (Supplementary material Appendix 1). Most of the studies were conducted in North America $(n=113)$ and Europe $(n=57)$, two geographic areas largely characterized by modest species richness (Barthlott et al. 1999). Of the studies conducted in North America and Europe, $22 \%$ involved endemic species. In contrast, of the studies conducted in all other geographic areas, $44 \%$ involved endemics. The percentage of endemic and non-endemic species studied was similar in tropical and non-tropical regions of the world $\left(\mathrm{X}^{2}=0.1665, \mathrm{p}=0.68\right)$.

\section{Self-compatibility}

Fifty-eight percent of the species in our data set were self-compatible, and the frequency of self-compatibility did not differ between endemics and non-endemics $\left(X_{1}^{2}=0.07, p=0.78\right)$. However, the PICs analysis detected an association between self-compatibility and endemism; in all six instances where both self-incompatibility and endemism change in the phylogeny, a transition towards endemism was accompanied by a transition towards self-compatibility $(p=0.031)$.

\section{Location of pollen limitation studies of endemics}

Endemic species were studied in regions with higher species richness than non-endemics (2040 sp./10 $000 \mathrm{~km}^{2}$ vs 1350 sp./10 $\left.000 \mathrm{~km}^{2} ; X^{2}{ }_{1}=33.03, \mathrm{p}<0.0001\right)$. Furthermore, $49 \%$ of studies conducted in regions of high biodiversity (i.e. above the median species richness) involved endemics whereas only $17 \%$ of the studies conducted in regions with low diversity (i.e. below the median species richness) involved endemics. These patterns are as expected, given the higher prevalence of endemism in species rich regions (Myers et al. 2000). 


\section{Pollen limitation: the effects of endemism and self-compatibility}

Overall, the plants in our data set were significantly pollen limited $(\mathrm{t}=10.8, \mathrm{n}=294, \mathrm{p}<0.0001)$, with an average pollen limitation effect size (ln R) of 0.59 ! 0.93 (range: -1.59 to 4.28 ). This is equivalent to 1.80 -times higher fruit set after supplemental pollination relative to natural pollen receipt.

In the two-way ANOVA, we found that self-incompatible species were more pollen limited than self-compatible ones (ln R: 0.90 【 0.09 vs 0.45 ! 0.07; $F_{1,290}=14.9, p=0.0001$ ), a result consistent with previous studies (Knight et al. 2005). We also found that endemic species were more pollen limited than non-endemic species ( 0.82 ! 0.10 vs 0.53 ! 0.06 ; $\left.\mathrm{F}_{1,290}=5.88, \mathrm{p}=0.016\right)$. This difference is not directly attributable to higher pollen limitation in tropical regions (Larson and Barrett 2000) because the percentage of endemic and non-endemic species included did not differ between tropical and non-tropical regions. The interaction between self-compatibility and endemism was not significant $\left(\mathrm{F}_{1,290}=1.32\right.$, $p=0.25)$, indicating that each factor acts additively. Within endemic species there was no significant effect of local population size on mean pollen limitation effect size (small vs large populations: 0.79 ! 0.15 vs 0.74 ! $0.14 ; \mathrm{F}_{1,89}=5.88$, $\mathrm{p}=0.81)$.

When we controlled for phylogenetic relatedness we found similar effects of self-compatibility on pollen limitation. Self-incompatible clades were far more pollen limited than their self-compatible relatives $(\mathrm{W}=424.5, \mathrm{DF}=53$, $p<0.0001$ ). However, no consistent divergence in pollen limitation was found between endemics and non-endemics in the complete data set $(\mathrm{W}=50.0, \mathrm{DF}=47, \mathrm{p}=0.34)$ or when the analysis was restricted to self-incompatible species $(\mathrm{W}=-10.0, \mathrm{DF}=23, \mathrm{p}=0.39)$.

\section{Pollen limitation and regional species richness: is the relationship similar for endemic and non-endemic species?}

For the self-incompatible species, pollen limitation increased with regional species richness, but the strength and shape differed depending on endemism. Endemic species showed an accelerating relationship between regional species richness and pollen limitation (non-parametric effect: $\mathrm{F}_{1,3}=5.97$, $p=0.002$; Fig. 2A). Whereas, the relationship for nonendemic species was best described by a positive linear relationship $\left(\mathrm{F}_{1,84}=10.76, \mathrm{p}=0.0015\right)$ and there was no significant non-linear contribution (non-parametric effect: $\mathrm{F}_{1,3}=0.83, \mathrm{p}=0.48$; Fig. 2B). Not surprisingly, when selfcompatible species were considered, pollen limitation did not change with regional species richness either in endemic (Fig. 2C) or non-endemic species (Fig. 2D) ( $p>0.05$ for the non-parametric and linear effects in both models).

The PICs analysis conducted for self-incompatible species supported the findings of the TIPS analyses. Pollen limitation increased with regional species richness more strongly in endemic ( $n=25$ contrasts, $F=7.06, p=0.014$; Fig. 3 ), than in non-endemic self-incompatible species $(n=63$ contrasts, $\mathrm{F}=0.72, \mathrm{p}=0.40$; Fig. 3). For self-compatible species, pollen limitation did not change with regional species richness in endemic ( $n=43$ contrasts, $F=0.417, p=0.83$ ) nor non-endemic species $(\mathrm{n}=72$ contrasts, $\mathrm{F}=0.037$, $\mathrm{p}=0.85)$.

\section{Discussion}

Our meta-analyses indicate that self-incompatible species in species-rich areas are particularly prone to pollen limitation, a result in agreement with Vamosi et al. (2006). Whether this result will pose an extinction threat to plant species depends on whether a species is present only in these diverse areas and thus will be globally affected by inadequate pollination. Our results indicate so because endemics are more prone to pollen limitation in general but particularly so in highly diverse regions. In the following paragraphs we discuss our main findings, highlighting those obtained when controlling for phylogenetic relatedness. We also suggest experimental studies to uncover the underlying mechanisms and consequences of variation in the diversity of flowering plant communities for reproductive success of endemic and widespread species.

\section{Pollen limitation and its relationship with regional species richness in endemic versus non-endemic species}

Our cross-species analyses of 287 species indicated that pollen limitation was higher for endemics, regardless of their local population sizes, suggesting that some of the intrinsic or ecological features characteristic of endemic species mentioned in the Introduction increase the magnitude of pollen limitation. However, the PICs analysis did not support the TIPS finding. The conflicting results from these two analyses may be attributable to the fact that the trait most strongly associated with propensity for pollen limitation - selfcompatibility - showed a strong phylogenetic signal in our data set and tended to be phylogenetically associated with endemism in the direction that would counter its effects. Specifically, in the context of our phylogeny, endemism was coupled with self-compatibility, a finding that could suggest evolution of self-compatibility as a mode of 'escape' from the limitation experienced by self-incompatible endemics. Within self-incompatible clades, however, there was still little independent effect of endemism on mean pollen limitation. This may be due to the fact that the difference in pollen limitation between both groups of species does not remain constant because the relationship between pollen limitation and regional species richness accelerated at the high end of diversity for endemics, but was linear for non-endemic species across the entire range (Fig. 2A-B).

In our analysis studies of pollen limitation in highly diverse regions included a higher frequency of endemics and, thus, endemic species likely contribute to the global pattern of increased pollen limitation with regional species richness (Vamosi et al. 2006) both by their increased representation in highly biodiverse areas (Myers et al. 2000) and by their stronger relationship between pollen limitation and species richness. Based on our findings, we expect lower pollen limitation and a weaker relationship with increasing local diversity in modestly diverse plant communities that 

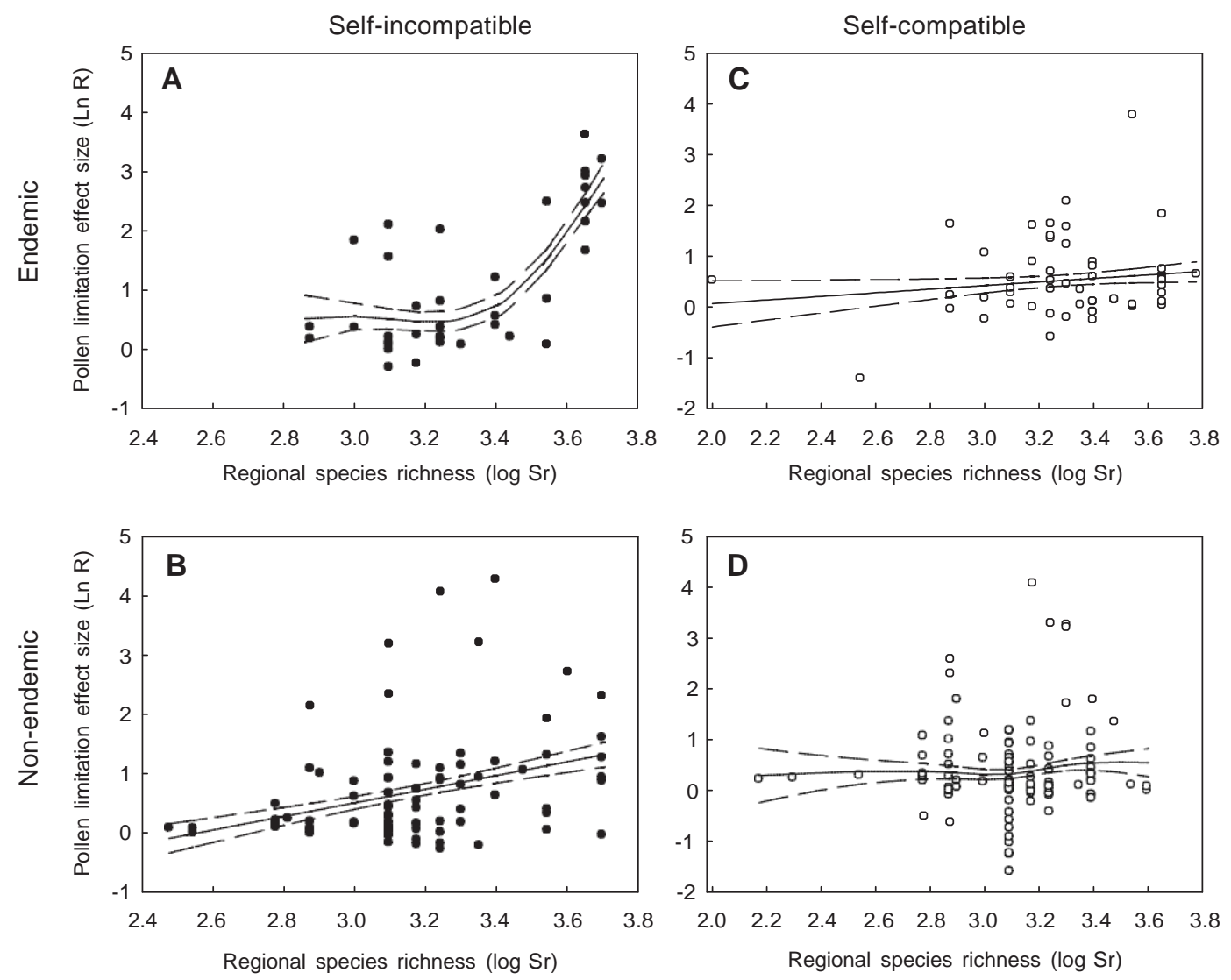

Figure 2. Non-parametric spline relationships between pollen limitation and regional species richness in endemic and non-endemic species by self-compatibility. In all graphs solid symbols denote self-incompatible species and open symbols self-compatible species. (A) Selfincompatible, endemic species, (B) self-incompatible, non-endemic species, (C) self-compatible, endemic species, and (D) self-compatible, non-endemic species. Dashed curves indicate I 1 SE of predicted values from 1000 bootstraps.

are poor in endemics (Motten 1986, Hegland and Totland 2008) than in highly diverse communities rich in endemics (Zjhra 2008).

Finally, we did not find a significant relationship between pollen limitation and diversity in self-compatible species (Fig. 2C-D). This result was not entirely unexpected given that many self-compatible species have an ability to autonomously self pollinate (Fenster and Martén-Rodríguez 2007) and thus are likely to show low levels of pollen limitation regardless of the diversity of their community or even benefit from the presence of co-occurring plants, i.e. through mitigation of an Allee effect (Feldman et al. 2004). However, we must note that current data do not account for the possibility that reproductive limitation may still occur via the quality of pollen received, an issue that should be explicitly addressed in the future.

\section{Future work: uncovering the mechanisms}

The patterns obtained by our meta-analysis at a global scale cannot identify the direct and indirect plant-plant pollinator interactions that may link species pollen limitation to the diversity of the local plant community in which they are imbedded, and that may account for the differential responses based on self-compatibility and endemism. Indeed, we cannot rule out the possibility that other environmental and historical factors related to differences in regional species richness on a global scale (Mutke and Barthlott 2005, Ohlemüller et al. 2008) may contribute to the variation in pollen limitation observed. We propose that further research in plant-pollinator interactions that is performed at the level of the community, accounts for quantity and quality of pollen transfer, and is conducted simultaneously on endemic and non-endemic species will clarify the mechanisms underlying the patterns outlined here.

\section{Understanding the functional relationship between diversity and pollen limitation}

Over the last few decades the central focus of research on plant-pollinator interactions has broadened from pair-wise interactions to community-wide assemblages (Bronstein 1994, Stanton 2003, Sargent and Ackerly 2008). Questions concerning the extent to which co-existing plants share pollinators (Waser et al. 1996), compete for or facilitate pollination (Levin and Anderson 1970, Feldman et al. 2004, Mitchell et al. 2009), and influence each others pollination sufficiency (Ashman et al. 2004) and ultimately reproductive success and species coexistence (Rathcke 1983, Ghazoul 2006, Sargent and Ackerly 2008) have come to the forefront.

It is at the community scale that a change along a species richness gradient in the plant-plant interaction from facilitation to competition for pollinator visits (Rathcke 1983) 


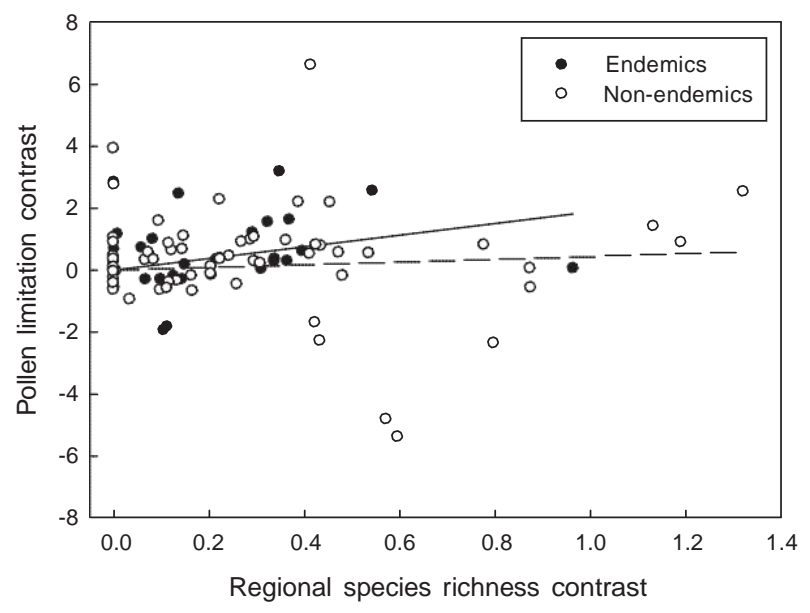

Figure 3. Standardized phylogenetic independent contrasts between sister groups in the phylogenetic tree obtained from PHYLOMATIC. Correlation analysis indicates that increases between sister groups in pollen limitation, measured as the effect size of fruit set, are correlated with increases between sister groups in community species richness only in self-incompatible endemic species. Along the $x$ axis are the absolute value of contrasts in regional species richness among sister groups, and along the $y$ axis are the corresponding pollen limitation contrasts. The figure shows the slope of the relaionship forced through the origin for self-incompatible endemic (solid symbols and straight line) and non-endemic (open symbols and dashed line) species.

could explain the increase in pollen limitation in species rich communities. Thus, a first step in understanding whether plant diversity affects pollination sufficiency via its influence on per capita pollinator visitation rate is to conduct pollinator observations on focal species across a wide range of diversity levels and determine whether visitation rates and magnitude of pollinator sharing varies with community diversity. While the effects of diversity on species-specific visitation rate have been studied in experimental arrays with a small number of species (Ghazoul 2006), the effects have been studied much less frequently in natural communities (Hegland and Totland 2008, Lázaro et al. 2008) or in highly diverse communities including endemics (Zjhra 2008). To be able to identify whether there is a threshold of species diversity at which the type of plant-plant interaction changes from facilitation to competition, studies will have to be conducted with larger ranges of diversity than has generally been attempted in the past.

\section{Why might endemic species be more susceptible to pollen limitation?}

Studies of indirect plant-plant pollinator interactions will need to include both endemic and non-endemic species in the same location to determine whether endemics within specific communities have characteristics that make them poorer competitors for pollinators (Karron 1987, Lavergne et al. 2005).

In addition to reduced visitation rates, increased heterospecific pollen transfer (reviewed by Morales and Traveset 2008) can lead to increased pollen limitation of plant reproduction through the quantity component, i.e. by reducing ovule fertilization. Heterospecific pollen deposition might be especially damaging for endemic species if they are neo-endemics and receive pollen from close taxonomically related species that not only clogs the stigma but germinates, interferes with conspecific pollen growth or even usurps ovules (Harder et al. 1992). Tropical endemic species with a long evolutionary history under similar environmental conditions might be exceptional in avoiding heterospecific pollen deposition (Zjhra 2008), although for these species extremely reduced visitation rates (Zjhra 2008) may still lead to stronger pollen limitation in tropical communities (Larson and Barrett 2000). Documentation of the pattern of conspecific and heterospecific pollen transfer with increasing community diversity, combined with hand-pollinations with various mixes of pollen applied in amounts similar to natural pollen loads will be needed to assess the effect of heterospecific pollen on seed production in both endemics and non-endemics and to determine whether these mechanisms could be responsible for the more extreme pollen limitation response to species richness exhibited by endemics.

Quality aspects of the conspecific pollen received have been given less attention than quantity aspects (Ashman et al. 2004, Aizen and Harder 2007) but they may explain the differences in pollen limitation between endemic and non-endemic species, and whether the apparent lack of association between pollen limitation and regional species richness for self-compatible species is real (Fig. 2C-D). Standard methods for assessing pollen limitation will be insufficient to disentangle the quality from quantity mechanisms, so methods that assess the relationships between natural pollen loads, pollen tube growth and seed production, combined with more rigorous tests of pollen limitation including self- and out cross-pollinations of emasculated flowers that are not excluded from pollinators in addition to the control open pollinated flowers (Motten 1986, Alonso 2005, Aizen and Harder 2007) will provide important insights.

We expect that endemics will suffer more reproductive limitation in the form of poor quality conspecific pollen received or lack of compatible mates (Byers 1995, Kirchner et al. 2005) than non-endemic species, provided endemics are more genetically depauperate as has often been found (Cole 2003). Furthermore, for self-compatible plants, we expect reduced pollen quality of pollen received with increasing diversity because recent studies show that the addition of a co-flowering species can lead to reduced outcrossing rates as a result of improper pollen transfer (Bell et al. 2005). Knowledge of the extent to which endemic self-compatible species are obligate selfers will also be of great value because it would support our prediction that one evolutionary trajectory is for endemics to evolve self-compatibility or autogamy as a means to reduce reliance on pollinators and persist in their characteristically highly diverse communities (results above, Kunin and Shmida 1997; but see Murray et al. 2002). Lastly, comparisons of quantity and quality of pollen transfer between endemics and non-endemics along a gradient of species diversity will clarify whether or not self-compatible species are affected by increasing diversity via a reduction in the quality of pollen received, and thus do actually respond to diversity as observed for self-incompatible species.

Altogether, if our finding that self-incompatible endemic species from biodiversity hotspots are at greatest risk of pollination failure is confirmed by the additional studies 
proposed above, then we suggest that increased effort should be devoted to the assessment of consequences for plant fecundity and population demography (Bevill and Louda 1999) in those areas. Such information will be useful in developing and prioritizing conservation strategies of wild flora, especially if the current scenario of pollinator decline, habitat fragmentation, and climate change effectively increases pollen limitation of plant reproduction (Knight et al. 2005, Memmott et al. 2007) as well as reduces the area of rare, localized, climatic conditions in which plant endemics prevail (Ohlemüller et al. 2008).

Acknowledgements - We thank Carlos M. Herrera for statistical advice and discussion, Marcelo Aizen, Diane Campbell and Jeff Ollerton for comments and discussion, Mónica Medrano, Sasha Rhode, Rachel Spigler and Nick M. Waser for critiques that improved the clarity of the manuscript. This work was supported by the National Center for Ecological Analysis and Synthesis, the Univ. of Pittsburgh, and the National Science Foundation [DEB 0449488 to TLA]; the Spanish Ministerio de Educación y Ciencia [CGL2006-01355/BOS] and Consejo Superior de Investigaciones Científicas [PIE200730I001] to CA; and a Natural Sciences and Engineering Research Council of Canada Discovery Grant to JCV.

\section{References}

Aizen, M. A. and Harder, L. D. 2007. Expanding the limits of the pollen-limitation concept: effects of pollen quantity and quality. - Ecology 88: 271-281.

Alonso, C. 2005. Pollination success across an elevation and sex ratio gradient in gynodioecious Daphne laureola. - Am. J. Bot. 92: 1264-1269.

Ashman, T.-L. et al. 2004. Pollen limitation of plant reproduction: ecological and evolutionary causes and consequences. Ecology 85: 2408-2421.

Barthlott, W. et al. 1999. Terminological and methodological aspects of the mapping and analysis of global biodiversity. Acta Bot. Fenn. 162: 103-110.

Bell, J. M. et al. 2005. Interspecific competition for pollination lowers seed production and outcrossing in Mimulus ringens. Ecology 86: 762-771.

Bevill, R. L. and Louda, S. M. 1999. Comparisons of related rare and common species in the study of plant rarity. - Conserv. Biol. 13: 493-498.

Bronstein, J. L. 1994. Our current understanding of mutualism. Q. Rev. Biol. 69: 31-51.

Byers, D. L. 1995. Pollen quantity and quality as explanations for low seed set in small populations exemplified by Eupatrium (Asteraceae). - Am. J. Bot. 82: 1000-1006.

Cole, C. T. 2003. Genetic variation in rare and common plants. Annu. Rev. Ecol. Evol. Syst. 34: 213-237.

Feldman, T. S. et al. 2004. When can two plant species facilitate each other's pollination? - Oikos 105: 197-207.

Fenster, C. B. and Martén-Rodríguez, S. 2007. Reproductive assurance and the evolution of pollination specialization. - Int. J. Plant Sci. 168: 215-228.

Freckleton, R. P. et al. 2002. Phylogenetic analysis and comparative data: a test and review of evidence. - Am. Nat. 160: 712-726.

Garland, T. et al. 1992. Procedures for the analysis of comparative data using phylogenetically independent contrasts. - Syst. Biol. 41: 18-32.

Ghazoul, J. 2006. Floral diversity and the facilitation of pollination. - J. Ecol. 94: 265-304.
Harder, L. D. et al. 1992. Unilateral incompatibility and the effects of interspecific pollination for Erythronium americanum and Erythronium albidum (Liliaceae). - Can. J. Bot. 71: 353-358.

Hegland, S. J. and Totland, O. 2008. Is the magnitude of pollen limitation in a plant community affected by pollinator visitation and plant species specialisation levels? - Oikos 117: 883-891.

Karron, J. D. 1987. The pollination ecology of co-occurring geographically restricted and widespread species of Astragalus (Fabaceae). - Biol. Conserv. 39: 179-193.

Kirchner, F. et al. 2005. Effects of local density on insect visitation and fertilization success in the narrow-endemic Centaurea corymbosa (Asteraceae). - Oikos 111: 130-142.

Klein, A.-M. et al. 2007. Importance of pollinators in changing landscapes for world crops. - Proc. R. Soc. Lond. B 274: 303-313.

Knight, T. M. et al. 2005. Pollen limitation of plant reproduction: pattern and process. - Annu. Rev. Ecol. Evol. Syst. 36: 467-497.

Kruckeberg, A. R. and Rabinowitz, D. 1985. Biological aspects of endemism in higher plants. - Annu. Rev. Ecol. Syst. 16: 447-479.

Kunin, W. E. and Shmida, A. 1997. Plant reproductive traits as a function of local, regional and global abundance. - Conserv. Biol. 11: 183-192.

Larson, B. M. H. and Barrett, S. C. H. 2000. A comparative analysis of pollen limitation in flowering plants. - Biol. J. Linn. Soc. 69: 503-520.

Lavergne, S. et al. 2004. The biology and ecology of narrow endemic and widespread plants: a comparative study of trait variation in 20 congeneric pairs. - Oikos 107: 505-518.

Lavergne, S. et al. 2005. Limitations on reproductive success in endemic Aquilegia viscosa (Ranunculaceae) relative to its widespread congener Aquilegia vulgaris: the interplay of herbivory and pollination. - Oecologia 142: 212-220.

Lázaro, A. et al. 2008. Co-flowering neighbors influence the diversity and identity of pollinator groups visiting plant species. Oikos 118: 691-702.

Levin, D. A. and Anderson, W. W. 1970. Competition for pollinators between simultaneously flowering species. - Am. Nat. 104: 455-467.

Memmott, J. et al. 2007. Global warming and the disruption of plant-pollinator interactions. - Ecol. Lett. 10: 710-717.

Mitchell, R. J. et al. 2009. New frontiers in competition for pollination. - Ann. Bot. 103: 1403-1413.

Morales, C. L. and Traveset, A. 2008. Interspecific pollen transfer: magnitude, prevalence and consequences for plant fitness. Crit. Rev. Plant Sci. 27: 221-238.

Motten, A. F. 1986. Pollination ecology of the spring wildflower community of a temperate deciduous forest. - Ecol. Monogr. 56: $21-42$.

Murray, B. R. et al. 2002. How plant life-history and ecological traits relate to species rarity and commonness at varying spatial scales. - Austral Ecol. 27: 291-310.

Mutke, J. and Barthlott, W. 2005. Patterns of vascular plant diversity at continental to global scales. - Biol. Skr. 55: 521-531.

Myers, N. et al. 2000. Biodiversity hotspots for conservation priorities. - Nature 403: 853-858.

Ohlemüller, R. et al. 2008. The coincidence of climatic and species rarity: high risk to small-range species from climate change. Biol. Lett. 4: 568-572.

Rabinowitz, D. 1981. Seven forms of rarity. - In: Synge, H (ed.), The biological aspects of rare plant conservation. Wiley, pp. 205-217.

Rathcke, B. J. 1983. Competition and facilitation among plants for pollination. - In: Jones, C. E. and Little, R. J. (eds), Handbook of experimental pollination biology. Van Nostrand Reinhold, pp. 305-329. 
Rymer, P. D. et al. 2005. Reproductive success and pollinator effectiveness differ in common and rare Persoonia species (Proteaceae). - Biol. Conserv. 123: 521-532.

Sargent, R. D. and Ackerly, D. D. 2008. Plant-pollinator interactions and the assembly of plant communities. - Trends Ecol. Evol. 23: 123-130.

Schluter, D. 1988. Estimating the form of natural selection on a quantitative trait. - Evolution 42: 849-861.

Stanton, M. L. 2003. Interacting guilds: moving beyond the pairwise perspective on mutualisms. - Am. Nat. 162: S10-S23.

Stevens, P. F. 2008. Angiosperm Phylogeny Website. Ver. 9. <www. mobot.org/MOBOT/research/APweb/>.

Thompson, J. D. et al. 2005. Ecological differentiation of Mediterranean endemic plants. - Taxon 54: 967-976.

Supplementary material (available online as Appendix O18026 at www.oikos.ekol.lu.se/appendix). Appendix 1.
Vamosi, J. C. et al. 2006. Pollination decays in biodiversity hotspots. Proc. Natl Acad. Sci. USA 103: 956-961.

Waser, N. M. et al. 1996. Generalization in pollination systems, and why it matters. - Ecology 77: 1043-1060.

Webb, C. O. et al. 2008. Phylocom: software for the analysis of phylogenetic community structure and trait evolution. Ver. 4.0.1. <www.phylodiversity.net/phylocom/>.

Wikström, N. et al. 2001. Evolution of the angiosperms: calibrating the family tree. - Proc. R. Soc. Lond. B 268: 2211-2220.

Wilcock, C. and Neiland, R. 2002. Pollination failure in plants: why it happens and when it matters? - Trends Plant Sci. 7: 270-277.

Zjhra, M. L. 2008. Facilitating sympatric species coexistence via pollinator partitioning in endemic tropical trees of Madagascar. Plant Syst. Evol. 271: 157-176. 